\title{
Stendhal, Il Laboratorio di sé. Corrispondenza, vol. I, vol. II, vol III
}

\section{Annalisa Bottacin}

\section{(2) OpenEdition}

1 Journals

\section{Edizione digitale}

URL: http://journals.openedition.org/studifrancesi/10657

DOI: $10.4000 /$ studifrancesi. 10657

ISSN: 2421-5856

\section{Editore}

Rosenberg \& Sellier

\section{Edizione cartacea}

Data di pubblicazione: 1 décembre 2017

Paginazione: 558-559

ISSN: 0039-2944

\section{Notizia bibliografica digitale}

Annalisa Bottacin, «Stendhal, II Laboratorio di sé. Corrispondenza, vol. I, vol. II, vol III», Studi Francesi [Online], 183 (LXI | III) | 2017, online dal 01 février 2018, consultato il 21 janvier 2021. URL: http:// journals.openedition.org/studifrancesi/10657 ; DOI: https://doi.org/10.4000/studifrancesi. 10657

Questo documento è stato generato automaticamente il 21 janvier 2021.

\section{(c) (i) (9)}

Studi Francesi è distribuita con Licenza Creative Commons Attribuzione - Non commerciale - Non opere derivate 4.0 Internazionale. 


\title{
Stendhal, Il Laboratorio di sé. Corrispondenza, vol. I, vol. II, vol III
}

\author{
Annalisa Bottacin
}

\section{NOTIZIA}

STENDHAL, Il Laboratorio di sé. Corrispondenza, vol. I (1800-1806), vol. II (1807-1812), vol III

(1813-1821), a cura di Vito Sorbello, Torino, Aragno, 2016, «Biblioteca Aragno», 787, 827, $673 \mathrm{pp}$.

Questa prima traduzione italiana della Correspondance di Stendhal, che si comporrà di sei volumi (ne sono usciti a oggi i primi tre), poteva essere vista come un evento straordinario sia per la qualità della materia che per l'importanza di un lavoro quanto mai atteso in Italia, paese prediletto dall'autore del Rouge. Victor Del Litto che con Pietro Paolo Trompeo aveva curato in ogni dettaglio la bella traduzione dei tre volumi dei Romanzi e Racconti, uscita a Firenze da Sansoni nel 1957, sovente mi ricordava quali e quante embûches si nascondessero nella pagina stendhaliana e con quanta attenzione furono selezionati i traduttori dell'opera. Perché la scrittura stendhaliana cela un «sospiro» che esce dalla pagina, un «sussurro» da leggersi quale necessario e affascinante passo per inoltrarsi nel testo, capirlo e diventarne partecipi. In effetti Mariella Di Maio nell'Introduzione al secondo volume della nuova traduzione dei Romanzi e Racconti, editi da Mondadori nel 2002 e magistralmente tradotti da Maurizio Cucchi, nota che «il testo stendhaliano è anche la scrittura marginale che non procede diretta allo scopo, ma si frena, si interrompe, esita, torna indietro, si corregge o promette di correggersi...» (p. 16). Se tutto ciò è ben visibile nel romanzo, lo è ancor più nelle lettere, scenario privato, intimistico; da quelle inviate alla prediletta sorella Pauline alle missive dirette agli amici più cari, tutte ricche di sensazioni, talvolta veri e propri récits con note di viaggio, relazioni su serate teatrali o incontri con cantanti, intellettuali o personaggi conosciuti in questo o in quel salotto. È un continuo dialogo tra sé e sé che si erge dalla pagina scritta, trasmessa al fruitore, tra un rimpianto, un impeto di gioia, un momento d'ira o di passione. In questa edizione compare anche la 
corrispondenza amministrativa e consolare, redatta da Henri Beyle nelle sue mansioni di funzionario dello stato francese; in numero maggiore quelle inviate ai suoi superiori dalle sede consolare di Civitavecchia; ma quali diversificazioni tra pubblico e privato! Un'aggiunta questa che, priva di una relazione introduttiva da parte del curatore che ne giustifichi la presenza, non convince e nemmeno se ne evidenzia la necessità.

2 La traduzione delle lettere è preceduta, solo nel primo volume, da una lunga introduzione, L'invenzione di sé (pp. vii-xxxviii) e da una Nota all'edizione (pp. xxxix-xlii) che portano la firma di Vito Sorbello, due scritti che avrebbero avuto maggior senso se redatti da uno specialista del grenoblese (presumo che in Italia, allorché Sorbello assunse tale compito - e reputo che il lavoro non sia durato solo qualche mese - erano ancora viventi Mariella Di Maio e Massimo Colesanti che avrebbero dato un apporto fondamentale a un'opera di tale spicco), oppure da qualche noto studioso francese dell'epistolario, quale Jacques Houbert, uno dei curatori dell'edizione della Correspondance Générale, edita da Champion (1997-99), cui dobbiamo anche il ritrovamento, nei diciassette anni che seguirono, di un gran numero di lettere redatte da Stendhal, per lo più al giurista fiorentino Vincenzo Salvagnoli, che non appaiono affatto in questa edizione. Tutto è esitante, sembra quasi che il curatore si appropri di qualcosa che non gli sia troppo familiare e lo versi sulla pagina bianca, dimenticando purtroppo di trovarsi in uno spazio letterario che pullula di critici, di studiosi internazionali che nel corso del tempo hanno sempre più analizzato l'opera e la figura di Stendhal. Vito Sorbello ne cita solo qualche nome: Pietro Paolo Trompeo, Bruno Pincherle, Carlo Cordié, Luigi Foscolo Benedetto e il più vicino a noi è quello di Leonardo Sciascia, scomparso nel 1989. E poi il nulla. Come non rilevare inoltre un'imperdonabile caduta sulle fonti delle lettere, in quanto viene citata quale prima edizione la Correspondance inédite curata da Romain Colomb? È ben noto, in effetti, che all'improvvisa morte di Stendhal, avvenuta a Parigi il 23 marzo 1842, il cugino ed esecutore testamentario, Romain Colomb, ritrovò tra le sue carte alcuni testi inediti, per lo più incompiuti, e nel timore che andassero perduti, li trasformò in una serie di presunte lettere inviategli dallo stesso Beyle, facendole precedere da un "cappello», quasi a giustificare questo curioso escamotage, e li inserì in uno degli ultimi volumi delle Euvres Complètes (Euvres Posthumes de Stendhal, Lettres à ses amis), che stava pubblicando per i tipi dell'editore Michel Lévy e cui diede il titolo di Correspondance inédite (Paris, 1855). In effetti non appaiono in alcuna raccolta della Correspondance. Un lettore che conosca solo approssimativamente Stendhal terrà certo conto di quanto esposto da Sorbello e riterrà quanto redatto degno di verità. Quest'edizione si basa, come il curatore riporta, sulla più recente pubblicazione, precedentemente citata, della Correspondance Générale, curata da Victor Del Litto con il supporto di E. Williamson, J. Houbert e M.-E. Slatkine; è accompagnata da poche note e pone comunque vari quesiti. Per quale ragione la frase stendhaliana viene allargata o ridotta a piacimento? Stendhal va rispettato, vi è sempre una ragione nelle sue scelte. Tale atto s'impone su quel «sospiro che viene dall'anima» di cui tanto parlava Del Litto, che fa scaturire intime tensioni, atti mancati, piccoli pensieri, in breve l'essenza di sé. Tradurre Stendhal ha indubbiamente le sue difficoltà, anche perché il suo stile non cambia di molto da una pagina autobiografica a quella di un romanzo, è uno stile che vede il fondo delle cose; l'impresa di chi si accinge a tradurlo presenta altri tranelli da quelli abituali che riguardano ogni traduzione di un'opera letteraria, come ricordava Colesanti; la traduzione è indubbiamente riscrittura, si avranno traduzioni più o meno fedeli, ma mai fuorvianti. «Reinterpretare» ha qui il valore di manomissione, di una 
presa di possesso; attenzione a non deformare o travisare eccessivamente il costrutto e il tono dell'originale, che poco si adatta a chi attraversa i sentieri che conducono in Stendhalie: essi sono scoscesi, impervi, ma l'attesa porta a paesaggi sublimi. È un cammino che richiede grande umiltà. 